\title{
Semidiscretization for time-delayed neural balance control
}

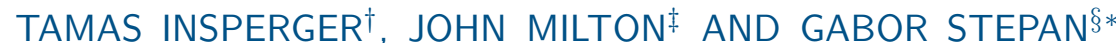

\begin{abstract}
The observation that time-delayed feedback can stabilize an inverted pendulum motivates the formulation of models of human balance control in terms of delay differential equations (DDEs). Recently the intermittent, digital-like nature of the neural feedback control of balance has become evident. Here, semidiscretization methods for DDEs are used to investigate an unstable dynamic system subjected to a digital controller in the context of a switching model for postural control. In addition to limit cycle and chaotic ("microchaos") oscillations, transiently stabilized balance states are possible even though both the open-loop and the closed-loop systems are globally unstable. The possibility that falls can be an intrinsic component of neural control of balance may provide new insights into how the risk of falling in the elderly can be minimized.
\end{abstract}

Key words. delay differential equations, switching, transient dynamics, microchaos, neural balance control

AMS subject classifications. 93C23, 93C $30,93 \mathrm{C} 23,92 \mathrm{C} 20$

1. Introduction. A fundamentally important question for human balance control, which up to now has received little attention, concerns how the differential equations for the neural control of balance should be formulated. To illustrate the problem, consider a controlled inverted pendulum whose small movements are described by the delay-differential equation (DDE) $[53,70]$

$$
\ddot{\theta}(t)-\omega_{\mathrm{n}}^{2} \theta(t)=-k_{\mathrm{p}} \theta(t-\tau)-k_{\mathrm{d}} \dot{\theta}(t-\tau)
$$

where $\theta$ is the vertical displacement angle, $\tau$ is the time delay, $\omega_{\mathrm{n}}$ is the natural angular frequency of small oscillations when the pendulum hangs downwards, and $k_{\mathrm{p}}$ and $k_{\mathrm{d}}$ are the proportional and the derivative gains. Since the left-hand side of (1.1) describes the motion of a Newtonian dynamical system, it evolves in continuous time. However, since the right-hand side describes a neuro-physiological system it has a distinctly digital quality reflecting, for example, the observation that spatially separated neurons communicate by discrete action potentials. Traditionally, mathematical models for the control of human balance $[2,34,35,43,50,66$, $70,74,78]$ and stick balancing $[22,33,42,53]$ have assumed that the feedback is a continuous and smooth function of time. Nonetheless, a number of experimental observations on human balance and movement suggest that the feedback exhibits a number of properties expected for digital control including the quantized nature of slow voluntary movements $[52,71,76,79]$, the intermittent character of corrective movements $[8,9,17,38,44,51,60]$ and the role of central refractory times $[72,73,75,79]$. Indeed, for certain balancing tasks, intermittent control works better than continuous control [46]. These observations have prompted many investigators to develop mathematical models which emphasize a role for event- and clock-driven intermittent

\footnotetext{
*Department of Applied Mechanics, Budapest University of Technology and Economics, Budapest 1521, Hungary (insperger@mm.bme.hu, stepan@mm.bme.hu), ${ }^{\ddagger}$ W. M. Keck Science Center, The Claremont Colleges, Claremont, CA 91711 (jmilton@kecksci.claremont.edu), §MTA-BME Research Group on Dynamics of Machines and Vehicles, Hungarian Academy of Sciences, Budapest 1521, Hungary.
} 
(a)

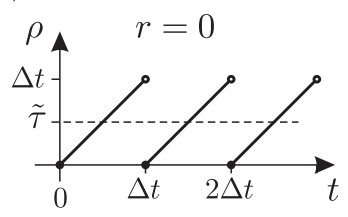

(b)

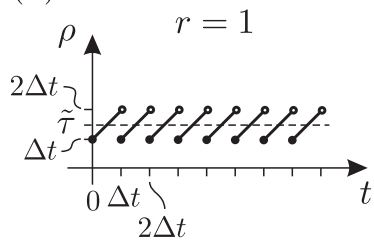

(c)

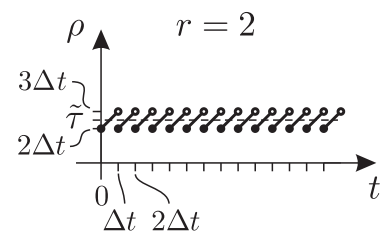

(d)

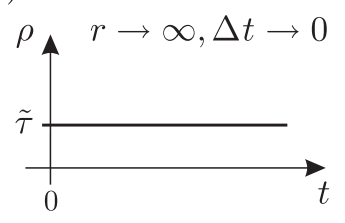

Figure 1. Time-varying delay in (1.2) for different values of $r$.

control strategies $[1,5,9,10,11,12,13,24,25,31,47,48]$. Here we consider the possibility that the neural feedback lies somewhere between these two extremes.

The recent development of semidiscretization methods for time-delay dynamical systems $[29,30]$ makes it possible to vary the feedback between discrete and continuous in a systematic manner. Based on analogies with the digital control of bipedal robots this approach suggests that an alternative model for balance control can be formulated as

$$
\ddot{\theta}(t)-\omega_{\mathrm{n}}^{2} \theta(t)=-k_{\mathrm{p}} \theta\left(t_{j}-r \Delta t\right)-k_{\mathrm{d}} \dot{\theta}\left(t_{j}-r \Delta t\right), \quad t \in\left[t_{j}, t_{j+1}\right), \quad j=0,1,2, \ldots,
$$

where the state variables are sampled at regular time instants $t_{j}=j \Delta t$ with $\Delta t$ being the sampling period $[29,30]$. In contrast to $(1.1)$, the delayed feedback term in (1.2) is piecewise constant in each sampling interval. Equation (1.2) can also be written as

$$
\ddot{\theta}(t)-\omega_{\mathrm{n}}^{2} \theta(t)=-k_{\mathrm{p}} \theta(t-\rho(t))-k_{\mathrm{d}} \dot{\theta}(t-\rho(t)),
$$

where $\rho(t)=r \Delta t+t-\operatorname{Int}(t / \Delta t)$ is a time-periodic delay and Int denotes the integer part function. In other words, for $t \in\left[t_{j}, t_{j+1}\right), \theta\left(t_{j}-r \Delta t\right)=\theta(t-\rho(t))$ [30]. The graph of the time-delay variation is shown in Figure 1 for different values of $r$. The average delay is

$$
\tilde{\tau}=\frac{1}{\Delta t} \int_{0}^{\Delta t} \rho(t) \mathrm{d} t=\left(r+\frac{1}{2}\right) \Delta t .
$$

It is well established that as $r \rightarrow \infty$ while $\Delta t \rightarrow 0$ such that

$$
\left(r+\frac{1}{2}\right) \Delta t=\tau
$$

holds, the solution of (1.2) approaches that of (1.1). In this sense, the semidiscrete model (1.2) provides a transition between intermittent and continuous-time representations of feedback control. The advantages of this semidiscretization of a DDE for numerical analysis stem from the fact that the Poincaré map has a simple finite dimensional representation, and hence (1.2) can be solved in each interval as a finite-order ordinary differential equation (ODE) in which the constant delayed terms are treated as a piecewise constant forcing in the ODEs. The important point for laboratory applications of the semidiscretization of DDEs is that the value of $r$ must be determined empirically by comparing observations with prediction.

Here we use the semidiscretization method to obtain insight into the surprising observation that transient balanced states lasting seconds to minutes can exist for the stabilization of an 
inverted pendulum with time delayed feedback even though the associated equilibria of both the closed-loop and the open-loop systems are globally unstable [31, 32]. We call a system globally unstable if all its fixed points and periodic and chaotic solutions are unstable, in other words, almost all of the solutions tend to infinity as $t \rightarrow \infty$. Complex, time-dependent and bounded states are observed experimentally for stick balancing at the fingertip [10, 11, 12, 55] and have been reproduced using computer simulations that involve either a deterministic $[31,32]$ or stochastic $[9,10]$ switching of time-delayed feedback control.

Recently, attention has focused on the possibility that switching dynamics for balance control during quiet standing are related to the presence of a sensory dead zone $[19,26,32$, $36,68,69]$. The fluctuations in $\theta$ during quiet standing are of the order of tenths of a degree and hence are below the threshold for the detection of movement for both the visual and vestibular system $[20,21,28]$. Proprioceptive sensors are able to detect changes on $|\theta|>0.05^{\circ}$ $[20,45]$ (see also section 6 of this paper). As a consequence, (1.1) becomes

$$
\ddot{\theta}(t)-\omega_{\mathrm{n}}^{2} \sin \theta(t)= \begin{cases}0 & \text { if } \theta(t-\tau)<\Pi, \\ f(\theta(t-\tau), \dot{\theta}(t-\tau), \ddot{\theta}(t-\tau)) & \text { otherwise },\end{cases}
$$

where $\Pi$ is the sensory threshold and we have taken into account the possibility that the feedback may depend not only on $\theta(t)$ and $\dot{\theta}(t)$ but also on the angular acceleration $\ddot{\theta}(t)$ (each of these contributions to the time-delayed feedback may be associated with a threshold). A sensory dead zone represents a strong small scale nonlinearity which has no effect on large scale stabilization of the linearized system, but can lead to complex dynamics on a small scale such as limit cycle oscillations and small amplitude chaotic oscillations, referred to as microchaos $[15,18,27]$. Transient chaotic behaviors associated with asymptotically stable equilibria are well recognized [14, 15, 39]. Here we demonstrate that equations of the form of (1.6) can generate long-lived, small-amplitude transient states referred to as transient microchaos even though the controlled system is globally unstable. The existence of these transient states implies that falling can be an intrinsic component of the neural control of balance!

We consider the model for postural sway during quiet standing proposed by Eurich and Milton [19]. In its simplest and dimensionless form this model is

$$
\dot{x}(t)=x(t)+f(x(t-\tau)),
$$

where the control input is a function of the delayed state $x(t-\tau)$ of the form

$$
f(x(t-\tau))= \begin{cases}C & \text { if } x(t-\tau)<-1, \\ 0 & \text { if }-1 \leq x(t-\tau) \leq 1, \\ -C & \text { if } x(t-\tau)>1\end{cases}
$$

where $C \geq 1$ is a constant and $\tau \leq \ln C$. Despite its simplicity this model incorporates three features of human balance control, namely an unstable upright position in the absence of feedback, stabilizing time-delayed feedback, and a sensory dead zone $[55,56]$. Although (1.7) is a simplified model of the Newtonian mechanics described by (1.6), it still presents the main feature of the inverted pendulum, namely instability occurs as an exponential fall (and not oscillatory). The solution of the linearized open loop system $\ddot{\theta}(t)-\omega_{n}^{2} \theta(t)=0$ is given as $\theta(t)=c_{1} \mathrm{e}^{\omega_{\mathrm{n}} t}+c_{2} \mathrm{e}^{-\omega_{\mathrm{n}} t}$ with $c_{1}$ and $c_{2}$ being constants depending on the initial conditions. For 
large $t$, the term $c_{2} \mathrm{e}^{-\omega_{\mathrm{n}} t}$ can be neglected and the solution tends to $c_{1} \mathrm{e}^{\omega_{\mathrm{n}} t}$, which is described by the first-order equation $\dot{\theta}(t)=\omega_{\mathrm{n}} \theta(t)$. After rescaling time, this gives the open-loop version of (1.7). In this sense, (1.7) can be considered as a projection to the unstable manifold of the open-loop system corresponding to (1.6).

The semidiscretized form of (1.7) is

$$
\dot{x}(t)=x(t)+f\left(x\left(t_{j}-r \Delta t\right)\right), \quad t \in\left[t_{j}, t_{j+1}\right),
$$

where

$$
f\left(x\left(t_{j}-r \Delta t\right)\right)= \begin{cases}C & \text { if } x\left(t_{j}-r \Delta t\right)<-1 \\ 0 & \text { if }-1 \leq x\left(t_{j}-r \Delta t\right) \leq 1 \\ -C & \text { if } x\left(t_{j}-r \Delta t\right)>1\end{cases}
$$

and $r$ and $\Delta t$ satisfy (1.5).

In Section 2 we show that when $r \rightarrow \infty$ the only possible stable solutions are three types of limit cycle oscillations. In Section 3 we show that when $r=0$ the limit cycle behaviors are replaced by three types of microchaotic solutions, each of which is associated with a transiently microchaotic solution. Numerical simulations are used in Section 4 to characterize the transition as $r$ changes from 0 to $\infty$ in terms of the power spectral density of the solutions. Finally in Section 5 we discuss the neurobiological significance of our findings.

2. Continuous-time case: $r \rightarrow \infty$. When $r \rightarrow \infty$, the solutions of (1.7) and (1.8) are the same and are equal to

$$
x(t)= \begin{cases}-C+\left[x\left(t_{0}\right)+C\right] \exp \left(t-t_{0}\right) & \text { if } x(t-\tau)<-1, \\ x\left(t_{0}\right) \exp \left(t-t_{0}\right) & \text { if }-1 \leq x(t-\tau) \leq 1, \\ C+\left[x\left(t_{0}\right)-C\right] \exp \left(t-t_{0}\right) & \text { if } x(t-\tau)>1 .\end{cases}
$$

As shown previously [19], the solutions of (1.7) depend only on two parameters: $\tau$ and $C$. There are no stable fixed points. Bounded solutions exist provided that $C \geq 1$ and $\tau \leq \ln C$. Within the parameter range for the bounded solutions, three distinct types of qualitatively different limit cycle oscillations exist (Figure 2(a): labeled O1, O2, and O3). In the region O1, two types of limit cycles coexist, one of which encircles 1 with $x>0$ and another one encircles -1 with $x<0$. In the region $\mathrm{O} 2$, the limit cycle oscillations have one breakpoint (a slope discontinuity) within the dead zone $x \in[-1,1]$ and one breakpoint outside the dead zone during one oscillation period. In the region $\mathrm{O} 3$, all of the breakpoints are outside the dead zone. The different oscillation types are shown in Figure 2(b),(c),(d). For a fixed pair $(C, \tau)$ in each region, there exist a series of limit cycles, which differ only in a phase shift depending on the initial condition. Note that the special symmetry of the oscillations are due to the symmetry of (2.1), which also explains the bistability in the region O1.

3. Discrete-time case: $r=0$. For the discrete counterpart of (1.7) the threshold condition is checked at discrete instants $t_{j}$. The solution of (1.8) with $r=0$ gives the discrete scalar map

$$
x\left(t_{j+1}\right)= \begin{cases}\exp (\Delta t) x\left(t_{j}\right)+C(\exp (\Delta t)-1) & \text { if } x\left(t_{j}\right)<-1 \\ \exp (\Delta t) x\left(t_{j}\right) & \text { if }-1 \leq x\left(t_{j}\right) \leq 1 \\ \exp (\Delta t) x\left(t_{j}\right)-C(\exp (\Delta t)-1) & \text { if } x\left(t_{j}\right)>1\end{cases}
$$



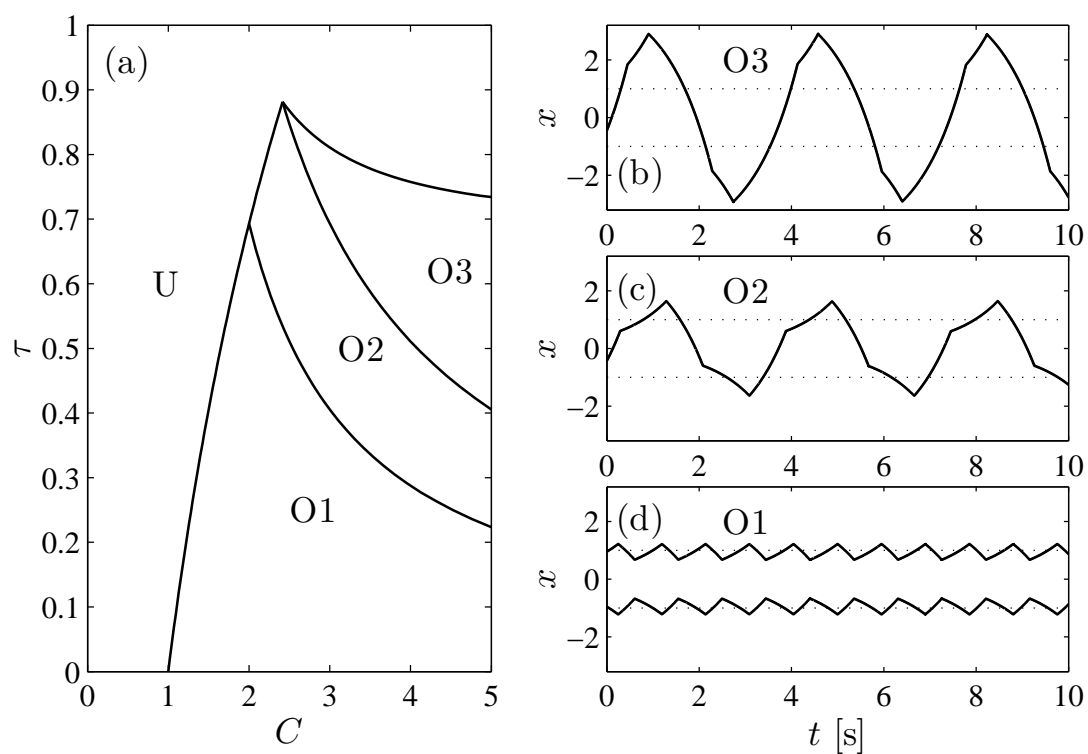

Figure 2. (a) Steady state behaviors of (1.7) observed as a function of $C$ and $\tau$. (b) Oscillation type O3. (c) Oscillation type O2. (d) Oscillation types 01 with positive and with negative initial state.

$\mathrm{U}$

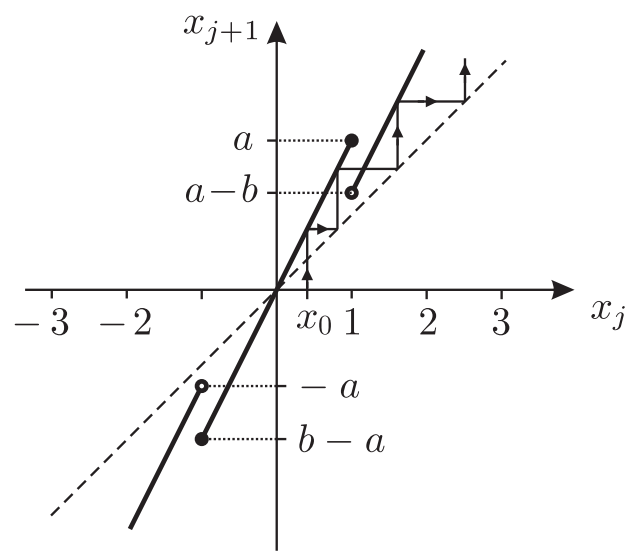

Figure 3. The map (3.2) with $b<a-1$ gives a globally unstable system.

It is convenient to rewrite this map in the form

$$
x\left(t_{j+1}\right)= \begin{cases}a x\left(t_{j}\right)+b & \text { if } x\left(t_{j}\right)<-1, \\ a x\left(t_{j}\right) & \text { if }-1 \leq x\left(t_{j}\right) \leq 1, \\ a x\left(t_{j}\right)-b & \text { if } x\left(t_{j}\right)>1,\end{cases}
$$

where $a=\exp (\Delta t)$ and $b=C(\exp (\Delta t)-1)$.

The steady state behavior of this system can be characterized by the parameters $a$ and $b$. The system is clearly globally unstable if $b<a-1$ (see Figure 3 ). Our goal is to demonstrate 
that chaotic and transient chaotic motions exist when $b>a-1$. We use the following definition of chaos [77].

Definition 1. Let $\mathcal{A}$ be a compact invariant and indecomposable set for $F: \mathbb{R} \rightarrow \mathbb{R}$ (i.e., if $x \in \mathcal{A}$ then for any integer $n \geq 0, F^{n}(x) \in \mathcal{A}$, and $\mathcal{A}$ cannot be written as a disjoint union of closed invariant subsets). Then $\mathcal{A}$ is said to be chaotic if

1. F has sensitive dependence on initial conditions on $\mathcal{A}$ (i.e., there exists a real number $\varepsilon>0$ such that for any $x \in \mathcal{A}$ and any $\delta>0$ there exists $y \in \mathcal{A}$ and an integer $n \geq 0$ such that $|x-y|<\delta$ and $\left.\left|F^{n}(x)-F^{n}(y)\right|>\varepsilon\right)$;

2. $F$ is topologically transitive on $\mathcal{A}$ (i.e., for any open sets $U \subset \mathcal{A}$ and $V \subset \mathcal{A}$ there exists an integer $n \geq 0$ such that $\left.F^{n}(U) \cap V \neq \emptyset\right)$.

It should be noted that if the solutions of (3.2) are bounded, then Theorem 6.4.1 and Remark 6.4.1 in [41] establish that they are ergodic. An alternative interpretation is that the positiveness of the Lyapunov exponent $(\Lambda=\ln (a)>0)$ guarantees sensitive dependence on initial conditions for (3.2). This follows from the fact that each of the branches are expanding since $a>1$. Thus, in order to show chaotic behavior, it is sufficient to demonstrate topological transitivity (see Appendix A for details).

The case when the signal under analysis appears chaotic up to certain time and then abruptly switches over into a nonchaotic behavior is called transient or metastable chaos $[14,39,81]$. A typical cause of transient chaos is that the set $\mathcal{A}$ is not invariant for $F$. In this case the trajectories can escape from $\mathcal{A}$ after a certain number of steps (see Figures $5(\mathrm{~b})$, $6(\mathrm{~b})$ and $7(\mathrm{~b}))$. The number of steps, at which a trajectory leaves the set $\mathcal{A}$, is called the lifetime or escape time [49]. The lifetime of transient chaos depends sensitively on initial conditions: nearby trajectories typically have drastically different lifetimes. Transient chaos is often characterized by the average of the lifetimes of trajectories initiated from random initial conditions. Exponential instability can be considered as a limit case of transient chaos with zero average lifetime.

Figure 4 summarizes the steady state behaviors of (3.2). There are three types of microchaos (MC) and three types of transient microchaos (TC) possible. There is a direct correspondence between the limit cycle solutions of (1.7) and the chaotic solutions of (3.2): $\mathrm{O} 1$ becomes MC1 and TC1, O2 becomes MC2 and TC2, and $\mathrm{O} 3$ becomes MC3 and TC3. In each case the transient microchaos exists in a parameter region in which the corresponding limit cycle is unstable. Thus the transient microchaotic solutions extend the region in parameter space in which balance can be maintained, albeit only transiently. In the next subsections, the different types of chaos are analyzed in detail in the discrete-time model of balancing.

3.1. MC1 and TC1. For $a-1<b<a$, the system presents microchaos of type MC1 if $b>a(a-1)$ (see Figure 5(a)). Apart from the unstable zero solution, there are two unstable fixed points indicated by $x= \pm x^{*}$. Two closed invariant and indecomposable attractive sets coexist in this case, $\mathcal{A}_{1}=[a-b, a]$ and $\mathcal{A}_{2}=[-a, b-a]$. Sensitive dependence on initial conditions can easily be shown by choosing $\varepsilon<b / 2$. Equivalently, the positiveness of the Lyapunov exponent $(\Lambda=\ln (a)>0)$ also implies sensitive dependence on initial conditions. Topological transitivity is established only for the parameter region given by the conditions 

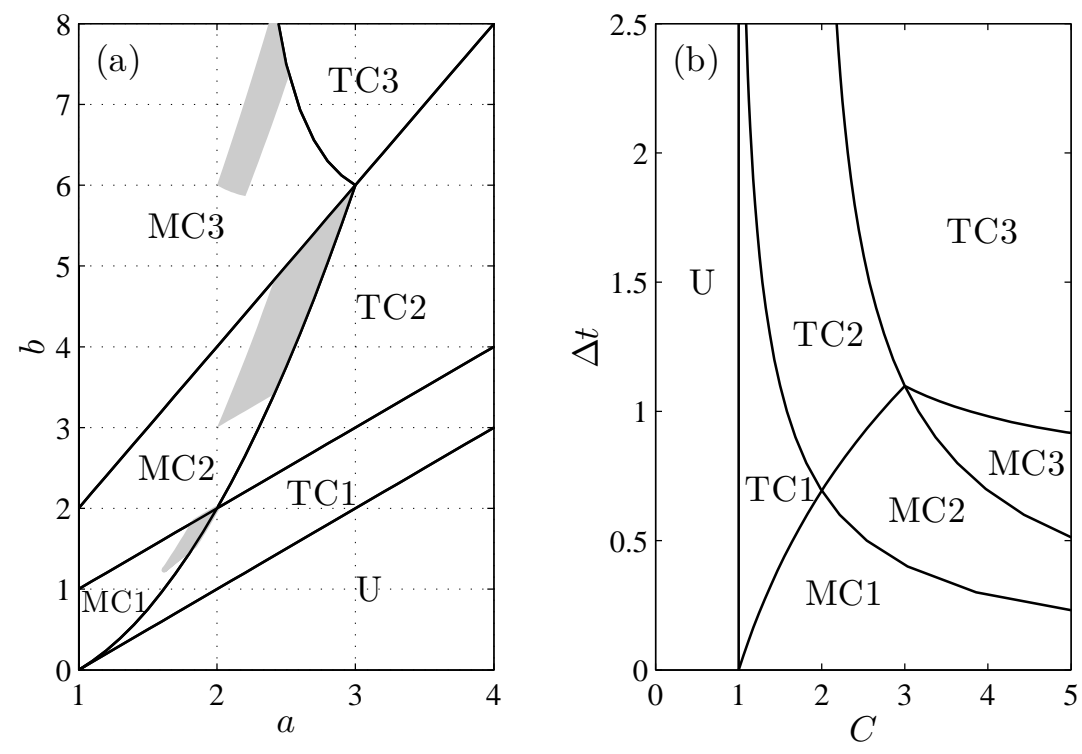

Figure 4. (a) Steady state behaviors of (3.2) observed as a function of a and b. (b) Steady state behaviors of (3.1) observed as a function of $C$ and $\Delta t$. Shading denotes the regions where topological transitivity is established.

$\mathrm{MC} 1$

(a)

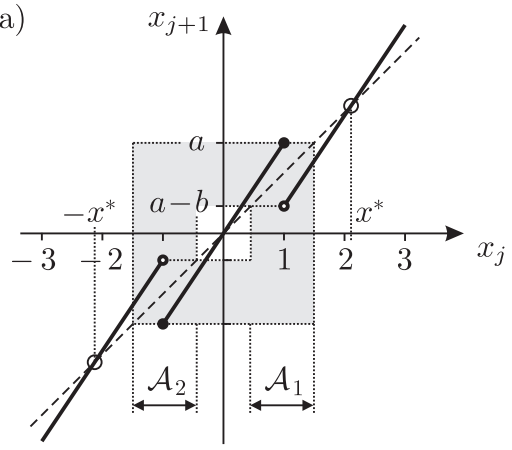

TC1

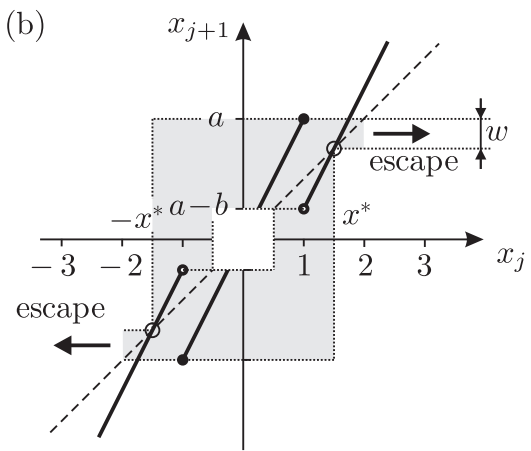

Figure 5. (a) The map (3.2) with $a-1<b<a$ and $b>a(a-1)$ gives a microchaos within the closed invariant sets $\mathcal{A}_{1}$ and $\mathcal{A}_{2}$ indicated by gray shading. The zero solution is unstable but the system is not globally unstable. (b) The map (3.2) with $a-1<b<a$ and $b<a(a-1)$ gives a transient microchaos: solutions persist temporarily within the gray region. The zero solution is unstable and the system is globally unstable.

(see Appendix A for details)

$$
b>a-\frac{1}{a}, \quad b>a(a-1), \quad b<\frac{a^{3}-1}{a+1}, \quad b<a-1,
$$

which is indicated by gray shading in the region of MC1 in Figure 4(a). It is conjectured that mixing can be shown in other domains of the parameter set $a-1<b<a, b>a(a-1)$ in a similar way. 

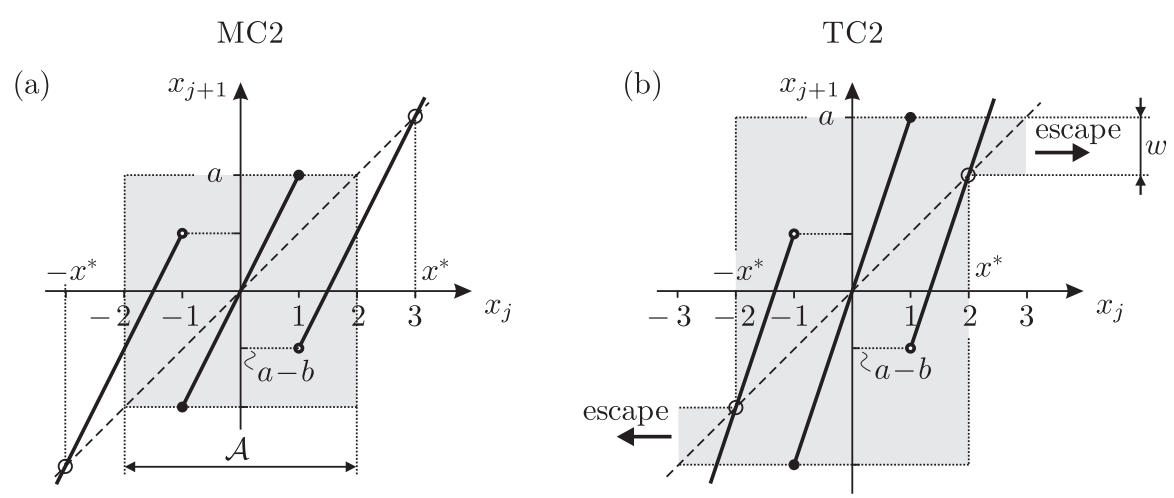

Figure 6. (a) The map (3.2) with $a<b<2 a$ and $b>a(a-1)$ gives a microchaos within the closed invariant set $\mathcal{A}$ indicated by gray shading. (b) The map (3.2) with $a<b<2 a$ and $b<a(a-1)$ gives a transient microchaos: solutions persist temporarily within the gray region.

The special case $b=a(a-1)$ represents a boundary crisis. If $a-1<b<a$ and $b<a(a-1)$, then the system presents transient microchaos (see Figure 5(b)). The length of the transients can be estimated by investigating the preimages of the escape windows. Clearly, the smaller the width of the escape window (denoted by $w$ in Figure 5(b)), the longer the average lifetime.

3.2. MC2 and TC2. If $a<b<2 a$, then the system presents microchaos of type MC2 if $b>a(a-1)$ (see Figure 6(a)). In this case, the closed invariant attractive set is $\mathcal{A}=$ $[-a, a]$. Since $\ln (a)>0$, the system has sensitive dependence on initial conditions. Topological transitivity is established for the parameter region given by the conditions (see Appendix A for details)

$$
b<a^{2}-1, \quad b>a+1,
$$

which is indicated by gray shading in the region of MC2 in Figure 4(a). It is conjectured that mixing can be shown in other domains of the parameter set $a<b<2 a, b>a(a-1)$ in a similar way.

The special case $b=a(a-1)$ represents a boundary crisis. If $a<b<2 a$ and $b<a(a-1)$, then the system presents transient microchaos (see Figure 6(b)). The width of the escape window is denoted by $w$ in Figure 6(b).

3.3. MC3 and TC3. If $b>2 a$, then the system presents microchaos of type MC3 if $b<a(a-1) /(a-2)$ (see Figure 7(a)). In this case, the closed invariant attractive set is $\mathcal{A}=$ $[a-b, b-a]$. Again, since $\ln (a)>0$, the system has sensitive dependence on initial conditions. Topological transitivity is established for the parameter region given by the conditions (see Appendix A for details)

$$
b>\frac{a^{2}+a}{a-1}, \quad b>a^{2}+1, \quad b<a^{2}+a,
$$

which is indicated by gray shading in the region of MC3 in Figure 4(a). It is conjectured that mixing can be shown in other domains of the parameter set $a<b<2 a, b<a(a-1) /(a-2)$ in a similar way. 
MC3

(a)

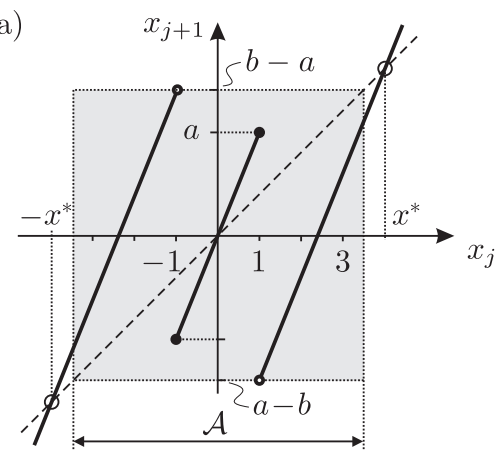

TC3

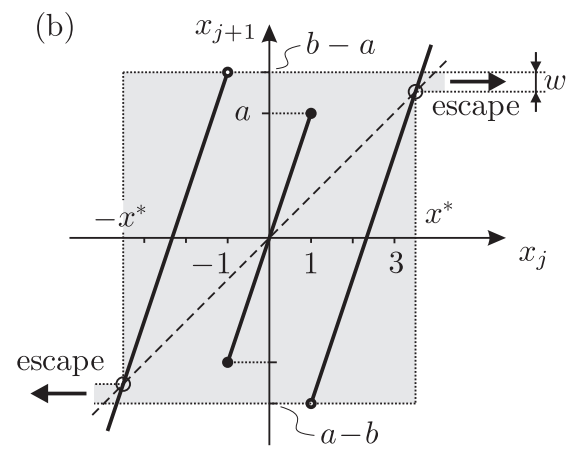

Figure 7. (a) The map (3.2) with $b>2 a$ and $b<a(a-1) /(a-2)$ gives a microchaos within the closed invariant set $\mathcal{A}$ indicated by gray shading. (b) The map (3.2) with $b>2 a$ and $b>a(a-1) /(a-2)$ gives $a$ transient microchaos: solutions persist temporarily within the gray region.
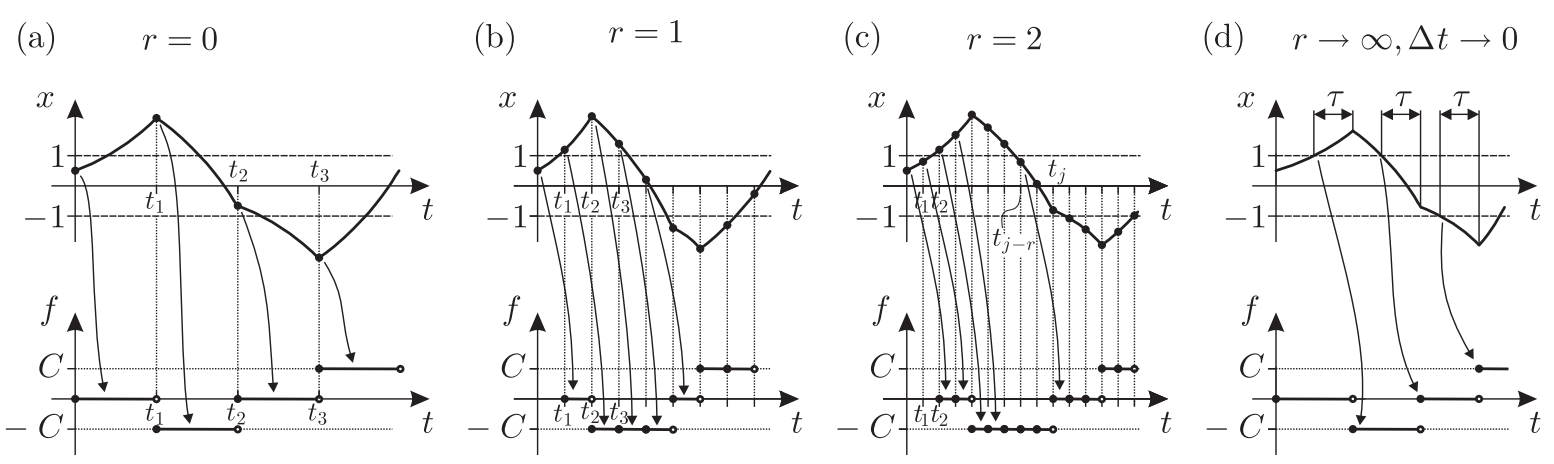

Figure 8. Switching mechanism of (1.8) for different $r$ values. The value of the feedback control at time interval $\left[t_{j}, t_{j+1}\right), j=0,1,2, \ldots$, is determined by the value of $x$ at time instant $t_{j-r}=t_{j}-r \Delta t$.

The special case $b=a(a-1) /(a-2)$ represents a boundary crisis. If $b>2 a$ and $b>$ $a(a-1) /(a-2)$, then the system presents transient microchaos (see Figure 7(b)). The width of the escape window is denoted by $w$ in Figure $7(\mathrm{~b})$.

4. Semidiscretization: $0<r<\infty$. The transition between the continuous-time (infinite dimensional) model and the scalar discrete-time model can be established by means of the semidiscretization method of delayed systems [30]. The corresponding hybrid model is described by (1.8). By varying $r$ we are able to observe the transition between digital control $(r=0)$ and continuous (analogue) control $(r \rightarrow \infty)$. While for finite values of $r$, switching in the control may occur at discrete sampling instants $t_{j}=j \Delta t$ only $(j=0,1,2, \ldots)$, for the case $r \rightarrow \infty$, switching takes place continuously in time. Figure 8 shows the switching mechanism for different $r$ values.

When $0<r<\infty$ a mixing of solutions is presumed to occur as a consequence of the interplay between the delay $\tau=\left(r+\frac{1}{2}\right) \Delta t$, the discrete time instants $t_{j}=j \Delta t$ and the threshold crossings. Figure 9 demonstrates the effect of choosing two different initial conditions (actually, initial functions) on the solutions of (1.8) for different values of $r$. Although the 
two solutions are close for a while, it happens at some discrete time instant $t_{j}$ that the two solutions will be on different sides of the threshold. When this happens, the solutions of (1.8) separate and diverge. Numerical solutions indicate that as $r$ increases towards $\infty$ it takes longer and longer for this phenomena to occur. Figure 10 shows the average separation time as function of $r$. One thousand uniformly distributed initial values $\left(x_{0}\right)$ were taken over the interval $[-1,1]$, each one was associated with a perturbed initial value $\tilde{x}_{0}=x_{0}-0.1$ and the separation time $t_{\text {sep }}=n \Delta t$ was recorded when the two solutions satisfied $\left|x_{n}-\tilde{x}_{n}\right|>2$. This concept corresponds to the sensitive dependence on initial conditions in Definition 1 with $\varepsilon=2$ and $\delta=0.1$. The numerical investigation confirms that the average separation time increases with $r$. When $r \rightarrow \infty$, the effect of choosing two 'nearby' initial functions is a phase shift between the limit cycle oscillations in $\mathrm{O} 2$ and $\mathrm{O} 3$, and also in $\mathrm{O} 1$ provided that no switch occurs between the coexistent oscillators.

Note that, in contrast with the discrete-time model $(r=0)$, the continuous-time model $(r \rightarrow \infty)$ does not have sensitive dependence on initial conditions, i.e., one can always give a number $\delta>0$ to any real number $\varepsilon$ such that for any two initial conditions $x_{0}$ and $\tilde{x}_{0}$ satisfying $\left|x_{0}-\tilde{x}_{0}\right|<\delta$ the corresponding solutions satisfy

$$
\max _{t \in[0, \infty)}(x(t)-\tilde{x}(t))<\varepsilon .
$$

This can be seen by means of the piecewise exponential solutions of (1.7) and the boundedness of the domain of attraction of the periodic solutions.

The transition $r \rightarrow \infty$ can also be explained by the Lyapunov exponent $\Lambda=\ln (a)=\Delta t$. As $r \rightarrow \infty, \Delta t \rightarrow 0$ since $\left(r+\frac{1}{2}\right) \Delta t=\tau=$ const. Consequently, the Lyapunov exponent tends to 0 , which explains the above explanation from a different aspect.

A convenient way to follow the changes in dynamics as $r$ increases is to monitor the changes in the power spectral density (PSD) of the solutions for each value of $r$ (see Figure 11). For the continuous-time case $(r=\infty)$ the PSD shows a principal peak at the frequency of the limit cycle oscillation and a number of peaks corresponding to its harmonics. As $r$ decreases, these peaks in the PSD disappear one by one. Figure 12 shows the survival times as $r \rightarrow 0$. Transiently bounded, microchaotic solutions exist in the parameter regions between the stability boundary for the dynamics of the continuous-time dynamical system and those for the discrete-time $(r=0)$ dynamical system.

We anticipate that the mixing phenomenon introduced by threshold crossings will also arise in higher-order DDEs that have been semidiscretized $(r<\infty)$. Indeed the transition between two attractors in DDEs typically takes multiple steps because any solutions with close initial conditions can be on opposite sides of the boundary between the two attractors $[23,59,63]$. The duration for the transiently stabilized state observed for (1.8) is an order of magnitude shorter than observed for (1.6) [31, 54]. Presumably, as the number of thresholds to be negotiated increases (e.g., one each for $\theta, \dot{\theta}, \ddot{\theta}$ ), the duration of the transiently stabilized state becomes longer and longer.

5. Neurobiological significance. Our observations provide insights into two questions concerning neural balance control that, up to now, have puzzled computational neuroscientists. First, continuous $(r \rightarrow \infty)$ DDE models for balance control with switching typically emphasize the occurrence of periodic limit cycle-type oscillations $[19,36]$. However, spectral analysis of 

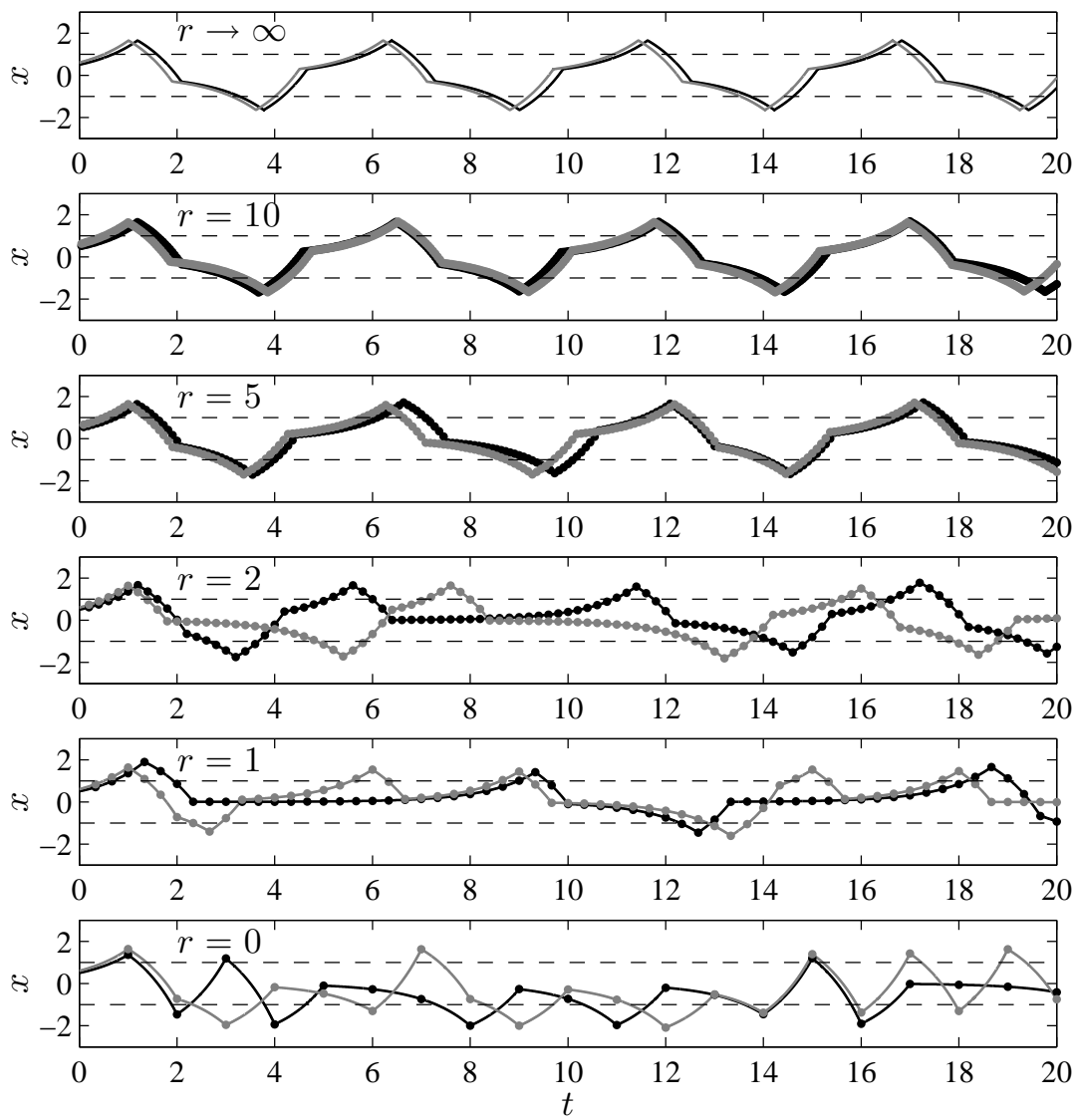

Figure 9. Demonstration of sensitivity on the initial conditions for different $r$ values. $C=3, \tau=0.5$, initial functions $x_{0}(s) \equiv 0.5$ (black), $x_{0}(s) \equiv 0.6$ (gray), $s \in[-\tau, 0]$. Discrete-time instants, where switching may take place are denoted by dots. Dead zone thresholds are indicated by dashed lines. Separation starts when the two solutions are on different sides of a threshold.

the fluctuations in the center of pressure (COP) for postural sway and the fluctuations in $\theta$ for stick balancing at the fingertip do not typically reveal the anticipated signatures of an oscillation, namely the presence of peaks in the PSD corresponding to the period and its associated harmonics and possibly subharmonics [37]. A PSD without any characteristic peaks is exactly what occurs for microchaos (see Figure 11). The value of $r$ relevant for balance control can be estimated from $\tau$ and $\Delta t$. For postural sway, $\tau \approx 0.13 \mathrm{~s}$ [35] and for stick balancing, $\tau \approx 0.22 \mathrm{~s}[31]$. The frequency by which the nervous system can execute a series of discrete movements is limited by a central refractory period of $\approx 0.2-0.5 \mathrm{~s}[48,72,73]$ suggesting that $\Delta t \approx 0.2-0.5 \mathrm{~s}$. Since $r=\tau / \Delta t-1 / 2,0<r \leq 1$ and we anticipate that the fluctuations observed for human balance control are microchaotic. These observations reaffirm the suggestion by Yamada in 1995 that the COP fluctuations recorded during quiet standing are chaotic [80]. Preliminary observations suggest that the fluctuations observed for stick balancing may also be chaotic [16]. An advantage of making control more digital 


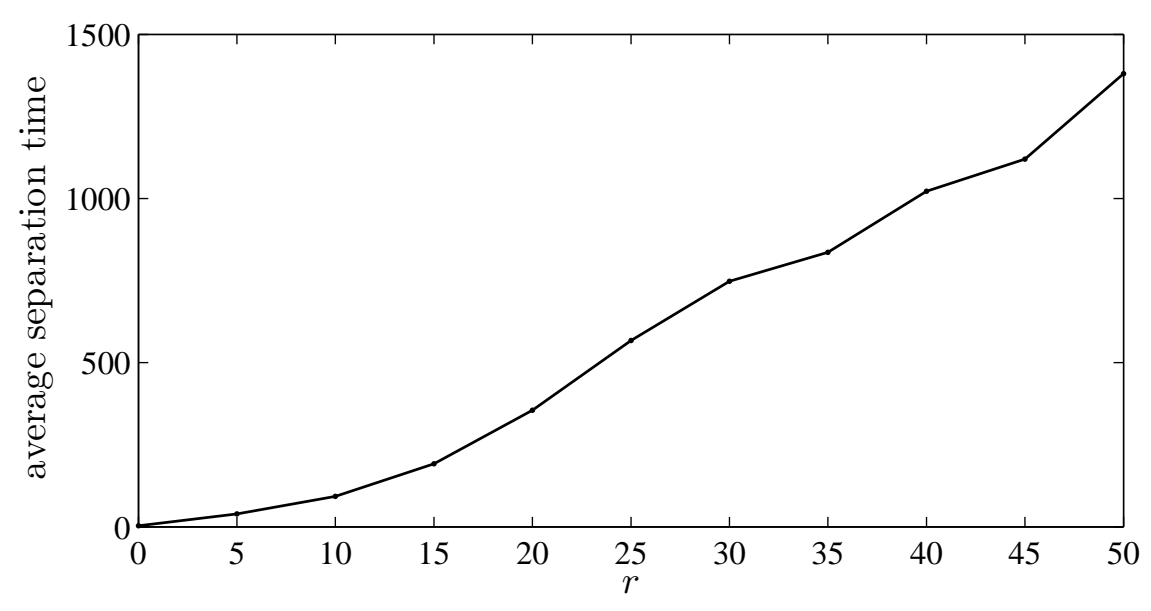

Figure 10. Average separation time of solutions with nearby initial conditions as function of $r$. C $=3$, $\tau=0.5$.

is to increase the range of parameter values for which balance can be maintained at least transiently.

The second puzzling observation concerns why a stick balanced at the fingertip eventually falls, even for the most skilled stick balancer. The suggestion that stick falls occur because of fatigue or distraction does not readily explain the observation that stick balancing is characterized by a Weibull-type survival function [10, 12]. Moreover, previous modeling studies have suggested that Weibull survival functions arise when the control gains are tuned to the unstable side of the stability boundary $[10,11]$. Both of these observations are consistent with the presence of transient microchaos. In other words it is possible that falls may not always be triggered by external agencies, but can be part of the intrinsic dynamics of the balance control as recently reported [64].

6. Discussion. Semidiscretization is a well-known technique for the analysis of partial differential equations that arise in the finite element analysis of solid structures and in computational fluid mechanics. In these PDEs, the spatial coordinate is discretized, while the temporal coordinate remains continuous. In contrast, the semidiscretization for DDEs has only been recently recognized and applications have focused on industrial problems, such as machine tool vibrations [30]. Here we suggest that semidiscretization methods very naturally arise in investigations of the control of movement and balance by the nervous system. In these situations, the continuous-time coordinate is associated with the dynamics of Newtonian mechanical systems which describe the muscle-skeletal systems and the discrete-time coordinate is associated with the neuro-muscular feedback controller. Here we have considered the simplest case of periodically sampled feedback control [46]. More generally we anticipate that the feedback control would be much more complex and likely contain both discrete and continuous components as well as a distribution of delays.

The role of a sensory dead zone on the control of postural sway during quiet standing remains an open question. It can be surprisingly difficult to distinguish experimentally between 

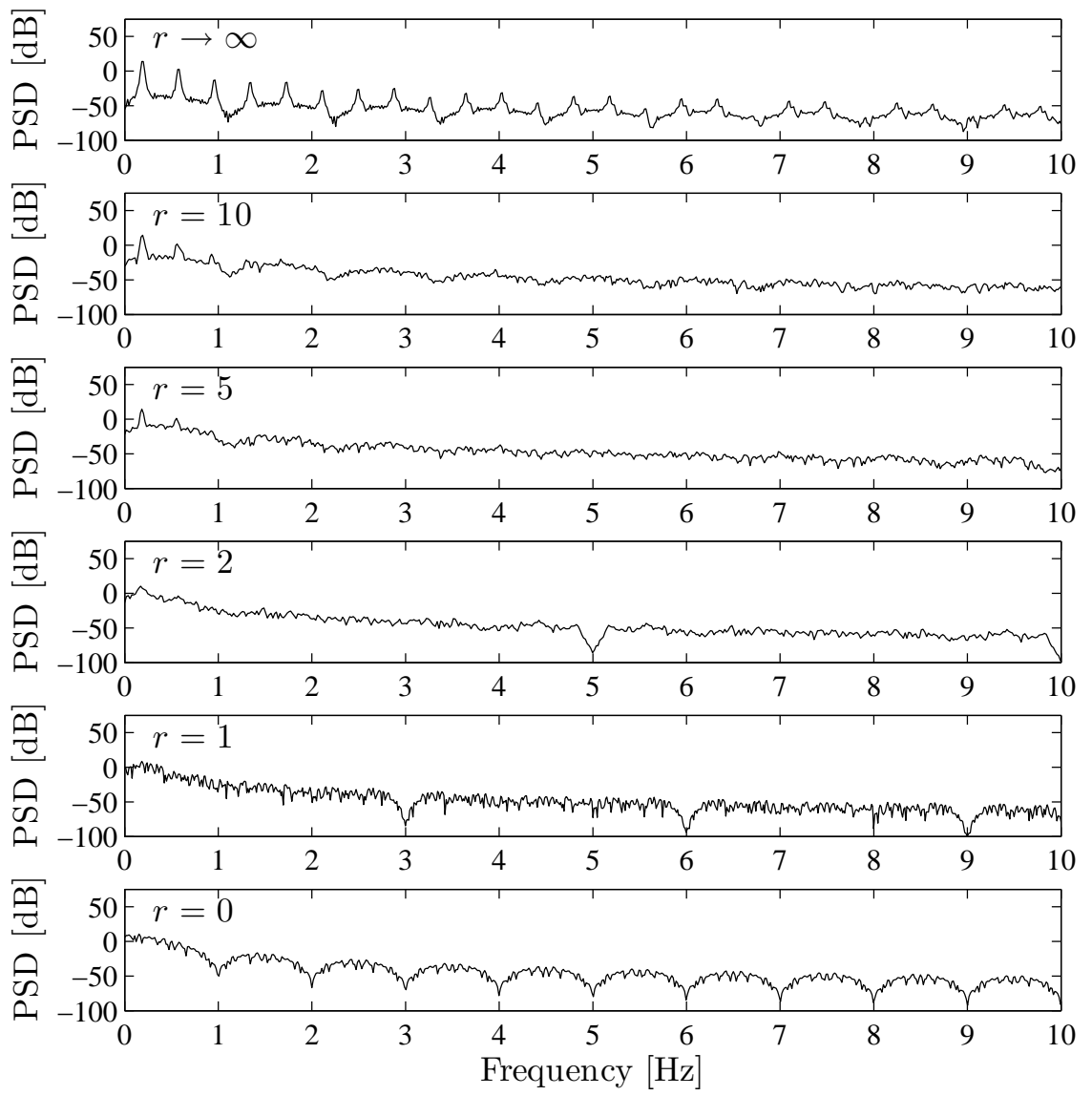

Figure 11. Power spectral densities for (1.8) with different $r . \tau=0.5, C=3$.

a nested control strategy that contains both open- and closed-loop control and continuous feedback control strategies using systems identification techniques [37, 74]. However, it is important to keep in mind that balance control during quiet standing is not synonymous with balance control during activities of daily living. As fluctuations in $\theta$ become larger, sensory inputs from the visual and vestibular systems become important and multijoint corrective movements are necessitated as manifested by the use of ankle-hip-step strategies [67]. Even greater challenges for balance control are imposed by locomotion since these activities require that the center of mass be displaced outside the base of support. It is reasonable to imagine that as the demands for balance control change, the discrete versus continuous nature of the neural feedback may change as well.

The prevention of falls in the elderly, and their accompanying mortality and morbidity, are major challenges faced by aging industrialized societies [7]. Surprisingly, there is little mathematical insight into why falls occur. Are falls simply the response to external perturbations, such as slips and trips or can falls occur as a result of the intrinsic dynamics of balance control regulation [64]? Although stochastic explanations for falling have been proposed [10, 55], our observations demonstrate that escape from a bounded time-dependent state can occur in a 
(a)

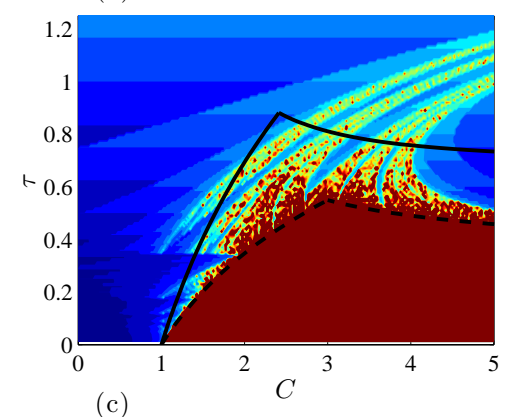

(c)

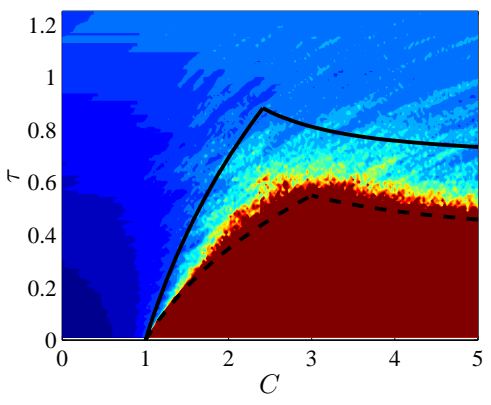

(b)

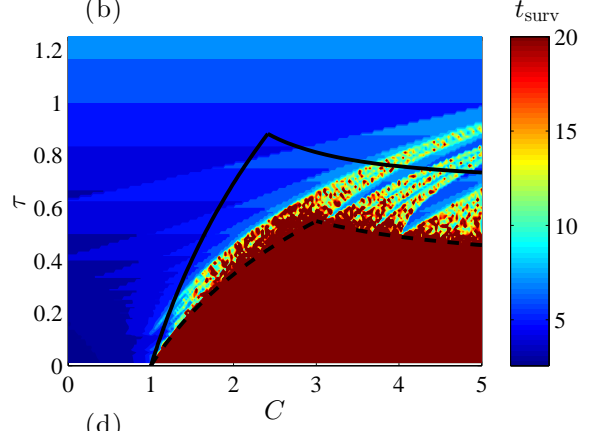

(d)

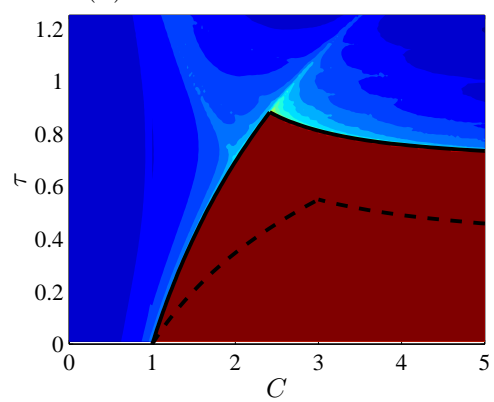

Figure 12. Color plots of the survival times $\left(t_{\text {surv }}\right)$ for (1.8) with $r=0$ for the initial conditions (a) $x(s) \equiv 0.5, s \in[-\tau, 0]$ and for (b) $x(s) \equiv 0.8, s \in[-\tau, 0]$ demonstrates the dependence on the choice of the initial function. Panel (c) shows the color plot of the average survival time for the ten different initial conditions distributed uniformly in $[0,1]$. Panel (d) shows the color plot of the survival time for (1.8) with $r=50$, which gives a good approximation of the continuous-time system (1.7) by means of semidiscretization. Dashed and continuous lines indicate, respectively, the boundaries of the region of permanently bounded solutions for the discrete-time system (1.8) with $r=0$ and for the continuous-time system (1.7).

deterministic model for balance control. The possible association of balance control with issues related to microchaos and transient microchaos may motivate a reevaluation of strategies based on the 'control of chaos' $[58,62,65]$ and stochastic resonance $[57,61]$ to minimize the risk of falling in the elderly. Thus we anticipate that our observations will motivate mathematicians and neuroscientists to use semidiscretized models to understand human balance control beyond quiet standing.

Acknowledgment. The authors acknowledge useful discussions with Jacques Bélair, Gábor Csernák, Michael C. Mackey, and Gergely Röst. This research project was partially supported by the Hungarian National Science Foundation under grant OTKA-K105433 (TI), the William R Kenan, Jr Charitable Trust (JM), the National Science Foundation (JM, NSF-1028970) and the Invitation Award to Distinguished Scientists by the Hungarian Academy of Sciences (JM). The research leading to these results has received funding from the European Research Council under the European Union's Seventh Framework Programme (FP7/2007-2013) / ERC Advanced grant agreement $\mathrm{N}^{\circ} 340889$. 
Appendix A. Establishing topological transitivity. Let us denote the map (3.2) by $F$. Topological transitivity of $F$ for the cases MC1, MC2, and MC3 are established in two steps $[3,4,6]$. First, we introduce the partition $\mathcal{P}=\left\{I_{i}\right\}_{i=1}^{n}$ of $\mathcal{A}$ such that $F$ is continuous on each interval $I_{i}$ and define the transition matrix $A=\left\{a_{i, j}\right\}$ induced by $F$ and $\mathcal{P}$ as

$$
a_{i, j}= \begin{cases}1 & \text { if } I_{j} \subset F\left(I_{i}\right), \\ 0 & \text { otherwise }\end{cases}
$$

(see, e.g., Section 7.3.1 in [3] or Definition 9.1.1. in page 174 in [6]). If the transition matrix $A$ is irreducible (i.e., for any $i, j$ there exists an integer $k$ such that $\left(A^{k}\right)_{i, j}$ are strictly positive), then the associated topological Markov chain is topologically transitive (see Proposition 7.4. in page 169 in [3]). Second, topological transitivity of the map $F$ is established by showing that for any open interval $U$ in $\mathcal{A}$, there exist an integer $m$ such that $F^{m}(U)$ contains a full interval $I_{i}$ of $\mathcal{P}$. According to Proposition 3.7 in [27], this step can easily be established if the map $F$ has enough expansion. To see this, let $\ell(V)$ denote the length of an open connected interval $V$. Assume that $U$ is an open interval in $\mathcal{A}$. Then $U$ has an open connected subset $U_{1} \subset U$ with length $L_{1}=\ell\left(U_{1}\right)$. Consider the set $F\left(U_{1}\right)$, which has an open connected subset $U_{2} \subset F\left(U_{1}\right)$ with length $L_{2}=\ell\left(U_{2}\right)$ such that $F$ is continuous on $U_{2}$ and $L_{2}>a L_{1} / 2$. Consider now the set $F\left(U_{2}\right)$ and take its open connected subset $U_{3} \subset F\left(U_{2}\right)$ such that $F$ is continuous on $U_{3}$ and $L_{3}>a L_{2} / 2$. Repeating this concept, a series $\left\{U_{m}\right\}_{m=1}^{\infty}$ of open connected intervals can be constructed with length

$$
L_{m}=\ell\left(U_{m}\right)=L_{1}\left(\frac{a}{2}\right)^{m-1}, \quad m=1,2, \ldots .
$$

If $a>2$, then there is an integer $m$, for which $L_{m}>\max _{i=1}^{n}\left(\ell\left(I_{i}\right)\right)$. Consequently, $F^{m}(U)$ contains a full interval $I_{i}$ of partition $\mathcal{P}$ (recall that $F$ is continuous on each interval $I_{i}$ ). Overall, if $a>2$, then it is sufficient to find a partition of $\mathcal{A}$, for which the associated transition matrix is irreducible. First, cases MC2 and MC3 are considered, then case MC1 is analyzed.

For the case $\mathrm{MC} 2$, the set $\mathcal{A}$ can be partitioned into the open intervals $I_{1}=(-a,-1)$, $I_{2}=(-1,1)$, and $I_{3}=(1, a)$. If

$$
\begin{aligned}
& F(-a)<-1, \\
& F(-1)>1,
\end{aligned}
$$

which directly imply condition (3.4), then the associated transition matrix reads

$$
A=\left(\begin{array}{lll}
0 & 1 & 0 \\
1 & 1 & 1 \\
0 & 1 & 0
\end{array}\right) .
$$

The graph of $F$ and a partition associated with the above conditions are shown in Figure 13(b). It can be seen that $A$ is irreducible since $A^{2}$ already has no zero entries. Consequently, the associated topological Markov chain is topologically transitive. Since the conditions (3.4) imply $a>2$, the map $F$ is topologically transitive in this parameter region. 
(a)

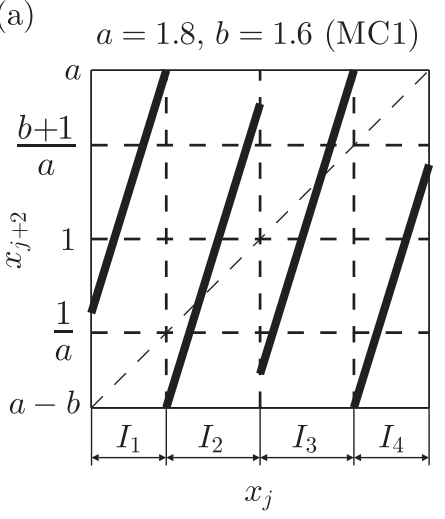

(b)

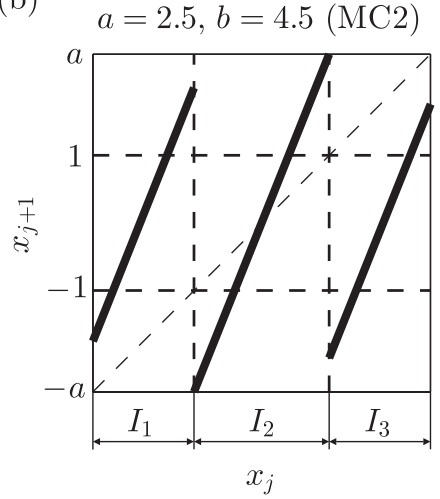

(c)

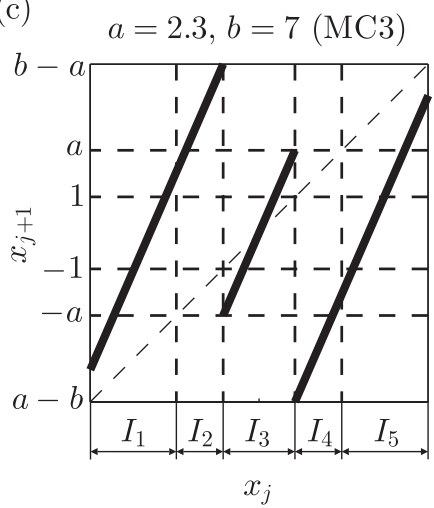

Figure 13. Partition of the invariant set $\mathcal{A}$ associated with (a) the map $G=F^{2}$ subjected to condition (3.3) for $M C 1$, (b) the map F subjected to condition (3.4) for $M C 2$, and (c) the map F subjected to condition (3.5) for $M C 3$.

For the case MC3, the set $\mathcal{A}$ can be partitioned into the open intervals $I_{1}=(a-b,-a)$, $I_{2}=(-a,-1), I_{3}=(-1,1), I_{4}=(1, a)$, and $I_{5}=(a, b-a)$. If

$$
\begin{gathered}
F(a-b)<-a, \\
F(-a)>1, \\
F(-a)<a,
\end{gathered}
$$

which directly imply condition (3.5), then the associated transition matrix reads

$$
A=\left(\begin{array}{lllll}
0 & 1 & 1 & 0 & 0 \\
0 & 0 & 0 & 0 & 1 \\
0 & 1 & 1 & 1 & 0 \\
1 & 0 & 0 & 0 & 0 \\
0 & 0 & 1 & 1 & 0
\end{array}\right)
$$

The graph of $F$ and a partition associated with the above conditions are shown in Figure 13(c). It can be seen that $A$ is irreducible since $A^{4}$ has no zero entries. Since the conditions (3.5) imply $a>2$, the map $F$ is topologically transitive in this parameter region.

For the case MC1, conditions (3.5) imply that $a<2$. Therefore, in this case, we consider the map $G=F^{2}$ with $x\left(t_{i}\right)>0$, which can be defined as

$$
x\left(t_{j+2}\right)= \begin{cases}a^{2} x\left(t_{j}\right) & \text { if } x\left(t_{j}\right) \leq 1 \text { and } a x\left(t_{j}\right) \leq 1, \\ a^{2} x\left(t_{j}\right)-b & \text { if } x\left(t_{j}\right) \leq 1 \text { and } a x\left(t_{j}\right)>1 \\ a^{2} x\left(t_{j}\right)-a b & \text { if } x\left(t_{j}\right)>1 \text { and } a x\left(t_{j}\right)-b \leq 1 \\ a^{2} x\left(t_{j}\right)-a b-b & \text { if } x\left(t_{j}\right)>1 \text { and } a x\left(t_{j}\right)-b>1\end{cases}
$$

For this map, the set $\mathcal{A}_{1}$ can be partitioned into the open intervals $I_{1}=(a-b, 1 / a), I_{2}=$ 
$(1 / a, 1), I_{3}=(1,(b+1) / a)$, and $I_{4}=((b+1) / a, a)$. If

$$
\begin{aligned}
G(a-b) & <1, \\
G(1 / a) & >1, \\
G(1) & <1, \\
G(a) & >1,
\end{aligned}
$$

which directly imply condition (3.3), then the associated transition matrix reads

$$
A=\left(\begin{array}{llll}
0 & 0 & 1 & 1 \\
1 & 1 & 0 & 0 \\
0 & 0 & 1 & 1 \\
1 & 1 & 0 & 0
\end{array}\right)
$$

The graph of $G$ and a partition associated with the above conditions are shown in Figure 13(a). It can be seen that $A$ is irreducible since $A^{2}$ has no zero entries. Since the conditions (3.5) imply $a>(1+\sqrt{5}) / 2 \approx 1.618$, the slope of $G$ is $a^{2}>2$. Consequently, the map $G$ is topologically transitive in the given parameter region, thus so is $F$.

\section{REFERENCES}

[1] Y. Asai, Y. Tanaka, K. Nomura, T. Nomura, M. Casidio and P. Morasso, A model of postural control in quiet standing: Robust compensation of delay-induced instability using intermittent activation of feedback control, PLoS ONE, 4 (2009), e6169.

[2] F. M. AtaY, The inverted pendulum using position feedback, Appl. Math. Lett., 12 (1999), pp. 51-56.

[3] L. Barreira AND C. VAlls, Dynamical Systems: An Introduction, Springer, Dordrecht, 2013

[4] J. BÉlair And P. Holmes, On linearly coupled relaxation oscillators, Quart. App. Math., 45 (1984), pp. 193-219.

[5] A. Bottaro, Y. Yasutake, T. Nomura, M. Casidio and P. Morasso, Bounded stability of the quiet standing posture: An intermittent control model, Human Mov. Sci., 27 (2008), pp. 473-495.

[6] A. Boyarsky And P. Gora, Laws of Chaos: Invariant Measures and Dynamical Systems in One Dimension, Birkäuser Boston, Boston, 1997.

[7] C. Bradley, Hospitalizations due to falls by older people, Australia, 2009-2010., Injury research and statistics series. no. 70, Australian Institute of Health and Welfare, Canberra, Australia, Cat. no. INJCAT 146.

[8] E. Burdet And T. E. Milner, Quantization of human motions and learning of accurate movements, Biol. Cybern., 78 (1998), pp. 307-318.

[9] J. L. Cabrera And J. G. Milton, On-off intermittency in a human balancing task, Phys. Rev. Lett., 89 (2002), 158702,

[10] J. L. Cabrera And J. G. Milton, On-off intermittency and survival times, Nonlinear Studies, 11 (2004), pp. 305-317.

[11] J. L. Cabrera, C. Luciani And J. Milton, Neural control on multiple time scales: Insights from human stick balancing, Cond. Mat. Phys., 9 (2006), pp. 373-383.

[12] J. L. Cabrera and J. G. Milton, Stick balancing, falls and Dragon Kings, Eur. Phys. J. Spec. Topics, 205 (2012), 231-241.

[13] C. C. Chow And J. J. Collins, Pinned polymer model of postural control, Phys. Rev. E, 52 (1995), pp. 907-912.

[14] G. Csernak and G. Stepan, Life expectancy of transient microchaotic behavior, J. Nonlinear Sci., 15 (2005), pp. 63-91. 
[15] G. Csernak and G. Stepan, Digital control source of chaotic motion, Int. J. Bifurcat. Chaos, 20 (2010), pp. $1365-1378$.

[16] G. Csernak, 2014, Personal communication.

[17] T. Cluff and R. Balasubramaniam, Motor learning characterized by changing Levy distributions, PLoS ONE, 4 (2009), e5988.

[18] E. Enikov and G. Stepan, Micro-chaotic motion of digitally controlled machines, J. Vib. Control, 4 (1998), pp. 427-443.

[19] C. W. Eurich And J. G. Milton, Noise-induced transitions in human postural sway, Phys. Rev. E, 54 (1996), pp. 6681-6684.

[20] R. Fitzpatrick and D. I. McCloskey, Proprioceptive, visual and vestibular thresholds for the perception of sway during standing in humans, J. Physiol., 478 (1994), pp. 173-186.

[21] R. Fitzpatrick, D. K. Rogers And D. I. MCCloskey, Stable human standing with lower-limb afferents providing the only sensory input, J. Physiol., 480 (1994), pp. 395-403.

[22] P. Foo, J. A. S. Kelso And G. C. De Guzman, Functional stabilization of fixed points: Human pole balancing using time to balance information, J. Exp. Psychol. Human Percept. Perform., 26 (2000), pp. 1281-1297.

[23] J. Foss, F. Moss And J. G. Milton, Noise, multistability and delayed recurrent loops, Phys. Rev. E, 55 (1997), pp. 4536-4543.

[24] P. Gawthrop, I. Loram, M. Lakie and H. Gollee, Intermittent control: a computational theory of human control, Biol. Cybern., 104 (2011), pp. 31-51.

[25] P. Gawthrop, K. Y. Lee, M. Halaki, and N. O'Dwywer, Human stick balancing: an intermittent control explanation, Biol. Cybern., 107 (2013), pp. 637-652.

[26] J. Guckenheimer, A robust hybrid stabilization strategy for equilibria, IEEE Trans. Automatic Control, 40 (1995), pp. 321-326.

[27] G. Haller and G. Stepan, Micro-chaos in digital control, J. Nonlinear Sci., 6 (1996), pp. 415-448.

[28] J. Hore, D. I. MCCloskey AND J. L. TAYlor, Task-dependent changes in gain of the reflex response to imperceptible perturbations of joint position in man, J. Physiol., 429 (1990), pp. 309-321.

[29] T. Insperger and G. Stepan, Semi-discretization method for delayed systems, Int. J. Numer. Meth. Engrg., 55 (2002), 503-518.

[30] T. Insperger and G. Stepan, Semi-discretization for time-delay systems, Springer, New York, 2011.

[31] T. Insperger and J. Milton, Sensory uncertainty and stick balancing at the fingertip, Biol. Cybern., 108 (2014), pp. 85-101.

[32] T. Insperger, J. Milton And G. Stepan, Acceleration feedback improves balancing against reflex delay, J. R. Soc. Interface, 36 (2013), pp. 2156-2163.

[33] V. K. Jirsa, P. Fink, P. Foo And J. A. S. Kelso, Parametric stabilization of biological coordination: a theoretical model, J. Biol. Phys., 26 (2000), pp. 85-112.

[34] H. G. Kang, L. Quach, W. Li And L. A. Lipshitz, Stiffness control of balance during dual task and prospective falls in older adults: The MOBILIZE Boston study, Gait Post., 38 (2013, pp. 757-763.

[35] T. Kiemel, Y. Zhang And J. J. JeKA, Identification of neural feedback for upright stance in humans: Stabilization rather than sway minimization, J. Neuroscience, 31 (2011), pp. 15144-15153.

[36] P. Kowalcyzk, G. Glendinning, M. Brown, G. Medrano-Cerda, H. Dallali and J. Shapiro, Modeling stick balancing using switched systems with linear feedback control, J. R. Soc. Interface, 9 (2012), pp. 234-245.

[37] P. Kowalcyzk, S. Nema, P. Glendinning, I. Loram and M. Brown, Auto-regressive moving average analysis of linear and discontinuous models of human balance during quiet standing, Chaos 24 (2014), 022101.

[38] H. I. Krebs, M. L. Aisen, B. T. Volpe And N. Hogan, Quantization of continuous arm movements in humans with brain injury, Proc. Natl. Acad. Sci. USA, 96 (1999), pp. 4645-4649.

[39] Y-C. Lai And T. Tel, Transient chaos: Complex dynamics on finite time scales, Springer, New York, 2011.

[40] M. Landry, S. A. Campbell, K. Morris and C. O. Aguilar, Dynamics of an inverted pendulum with delayed feedback control, SIAM J. Appl. Dyn. Sys. 4 (2005), pp. 333-351.

[41] A. LAsota AND M. C. MACKEy, Chaos, fractals and noise: Stochastic aspects of dynamics, SpringerVerlag, New York, 1994. 
[42] K. Y. Lee, M. O'Dwyer, M. Halaki and R. Smith, A new paradigm for human stick balancing: a suspended not inverted pendulum, Exp. Brain Res. 221 (2012), pp. 309-328.

[43] D. B. Lockhart And L. H. Ting, Optimal sensorimotor transformations for balance, Nat. Neurosci., 10 (2007), pp. 1329-1336.

[44] I. Loram, C. N. Maganaris And M. LAKIE, Human postural sway results from frequent, ballistic bias impulses by soleus and gastrocnemius, J. Physiol., 564 (2005), pp. 295-311.

[45] I. D. Loram, M. Lakie, I. Di Giulo and C. N. Maganaris, The consequences of short-range stiffness and fluctuating muscle activity for proprioception of postural joint rotations: The relevance to human standing, J. Neurophysiol., 102 (2009), pp. 460-474.

[46] I. D. Loram, H. Gollee, M. Lakie and P. J. Gawthrop, Human control of an inverted pendulum: Is continuous control necessary? is intermittent control effective? is intermittent control physiological?, J. Physiol., 589 (2011), pp. 307-324.

[47] I. D. Loram, C. van de Kamp, H. Gollee and P. J. Gawthrop, Identification of intermittent control in man and machine, J. R. Soc. Interface, 9 (2012), pp. 2070-2084.

[48] I. D. Loram, C. van de Kamp, M. Lakie, H. Gollee and P. J. Gawthrop, Does the motor system need intermittent control?, Exerc. Sport Sci. Rev., 42 (2014), pp. 117-125.

[49] M. C. Mackey and J. G. Milton, A deterministic approach to survival statistics, J. Math. Biol., 28 (1990), pp. 33-48.

[50] C. Maurer and R. J. Peterka, A new interpretation of spontaneous sway measures based on a simple model of human postural control, J. Neurophysiol., 93 (2005), pp. 89-100.

[51] R. C. Miall, D. J. Weir And R. F. Stein, Intermittency in human manual tracking tasks, J. Mot. Behav., 25 (1993), pp. 53-63.

[52] T. E. Milner AND M. M. IJAZ, The effect of accuracy constraints on three-dimensional movement kinematics, Neuroscience, 35 (1990), pp. 365-374.

[53] J. Milton, J. L. Cabrera, T. Ohira, S. Tajima, Y. Tonoskai, C. W. Eurich and S. A. CampBELL, The time-delayed, inverted pendulum: Implications for human balance control, Chaos, 19 (2009), 026110 .

[54] J. Milton, T. Insperger And G. Stepan, Human balance control: Dead zones, intermittency and micro-chaos. In: Mathematical approaches to biological systems: Networks, oscillations and collective phenomena (T. Ohira and T. Ozawa, eds), Springer, Japan, pp. 1-28.

[55] J. G. Milton, T. Ohira, J. L. Cabrera, R. M. Fraiser, J. B. Gyorffy, F. K. Ruiz, M. A. Strauss, E. C. Balch, P. J. Marin And J. L. Alexander, Balancing with vibration: A prelude for "drift-and-act" balance control, PLoS ONE, 4 (2009), e7427.

[56] J. Milton, J. Gyorffy, J. L. Cabrera And T. Ohira, Amplitude control of human postural sway using Achilles tendon vibration, 16th US National Congress of Theoretical and Applied Mechanics, State College. PA, USNCTAM2010-791 (2010).

[57] F. Moss And J. Milton, Balancing the unbalanced, Nature, 425 (2003), pp. 911-912.

[58] E. Ott, C. Grebogi And J. A. Yorke, Controlling chaos, Phys. Rev. Lett., 64(11) (1990), pp. 11961199.

[59] K. Pakdaman, C. Grotta-Ragazzo and C. P. Malta, Transient regime duration in continuous-time networks with delay, Phys. Rev. E, 58 (1998), pp. 3623-3627.

[60] F. Patzelt, M. Riegel, U. ERnst and K. Pawelzik, Self-organized critical noise amplification in human closed loop control, Front. Comput. Neurosci., 1 (2007), 4.

[61] A. A. Priplata, J. B. Niemi, J. D. Harry, L. A. Lipsitz And J. J. Collins, Vibrating insoles and balance control in elderly people, Lancet, 362 (2003), pp. 1123-1124.

[62] K. Pyragas, Continuous control of chaos by self-controlling feedback, Phys. Lett. A 170(60), (1992), pp.421-428.

[63] A. Quan, I. Osorio, T. Ohira And J. Milton, Vulnerability to paroxysmal oscillations in delayed neural networks: A basis for nocturnal frontal lobe epilepsy?, Chaos, 21 (2011), 047512.

[64] S. N. Robinovitch, F. Feldman, Y. Yang, R. Schonnop, P. M. Leung, T. Sarraf, J. Sims-Gould AND M. LOUGHLIn, Video capture of the circumstances of falls in elderly people residing in long term care: an observational study, Lancet, 381 (2013), pp. 47-54.

[65] E. Schöll AND H. G. Schuster, Handbook of Chaos Control, 2nd edition, John Wiley \& Sons, New York, 2008. 
[66] F. Schürer, Zur Theorie des Balanciereus, Math. Nachr. 1 (1948), pp. 295-331.

[67] A. Shumway-Cook And M. H. Woollacott, Motor Control: Theory and Applications, Second Edition, Lippincott, Williams \& Williams, New York, 2001.

[68] J. Sieber, Dynamics of delayed relay systems, Nonlinearity, 19 (2006), pp. 2489-2527.

[69] J. Sieber, P. Kowalczyk, S.J. Hogan And M. Di Bernardo, Dynamics of symmetric dynamical systems with delayed switching, J Vib. Control, 16 (2010), pp. 1111-1140.

[70] G. Stepan, Delay effects in the human sensory system during balancing, Philos. Trans. A Math. Phys. Eng. Sci., 367 (2009), pp. 1195-1212.

[71] A. B. VAlbo AND J. WessBerg, Organization of motor output in slow finger movements in man, J. Physiol., 469 (1993), pp. 673-691.

[72] C. van DE KAmp, P. J. GaWthrop AND I. D. LoRAm, Measuring the refractory period in a visual-manual pursuit task, Proc. Physiol. Soc., 23 (2011), PC89.

[73] C. van de Kamp, P. J. Gawthrop, H. Gollee and I. D. Loram, Refractoriness in sustained visuomanual control: Is the refractory duration intrinsic or does it depend on external system properties? PLoS Comp. Biol., 9 (2013), e1002843.

[74] H. van Der Kooij And E. De Vlugt, Postural responses evoked by platform perturbations are dominated by continuous feedback, J. Neurophysiol., 98 (2007), pp. 730-743.

[75] M. A. VINCE, The intermittency of control movements and the psychological refractory period, Br. J. Psychol. Gen. Sect., 38 (1948), pp. 149-157.

[76] C. vonHofsten, Structuring of early reaching movements: a longitudinal study, J. Mot. Behav., 23 (1991), pp. $280-292$.

[77] S. Wiggins, Chaotic Transport in Dynamical Systems, Springer-Verlag, New York, 1992.

[78] D. A. Winter, A. E. Patla, M. Ishac And K. Gielo-Perczak, Stiffness control of balance during quiet standing, J. Neurophysiol., 80 (1998), pp. 1211-1221.

[79] R. S. Woodward, The accuracy of voluntary movement, Tje Macmillan Company, New York, 1899.

[80] N. Yamada, Chaotic swaying of the upright posture, Hum. Mov. Sci., 14 (1995), pp. 711-726.

[81] J. A. Yorke And E. D. Yorke, Metastable chaos: the transition to sustained chaotic behavior in the Lorenz model, J. Stat. Phys., 21 (1979), pp. 263-277. 